GSA Data Repository 2016336

2

\title{
A carbonate-based proxy for sulfate-driven anaerobic oxidation of methane
}

\author{
Dong Feng ${ }^{*}$, Yongbo Peng ${ }^{*}$, Huiming Bao, Jörn Peckmann, Harry H. Roberts, and
}

Duofu Chen

\section{Materials}

Authigenic carbonates analyzed in this study came from three modern

hydrocarbon-seep provinces (Gulf of Mexico, Black Sea, and South China Sea) and six ancient hydrocarbon-seep deposits (Marmorito, Montepetra, and Ca' Piantè, northern Italy, Miocene; Knappton, SR4 deposit of the Lincoln Creek Formation, and Whiskey Creek, western Washington, USA, Eocene to Oligocene; Table DR1 and Table DR2). Specifically, authigenic carbonates from 12 seeps from the northern Gulf of Mexico (GOM) continental slope were selected. These carbonate rocks were collected from the late 1980 s to 2010 at water depths between $253 \mathrm{~m}$ and $2216 \mathrm{~m}$ (Roberts et al., 2010; Roberts and Feng, 2013; Feng et al., 2014, Feng and Roberts, unpublished data). Samples from the Black Sea were collected at locations on the Romanian (120 m depth) and Ukrainian (190 m depth) shelf and slope (Peckmann et al., 2001). Two samples from the South China Sea were described in Tong et al. (2013), and these samples were collected at a water depth of $769 \mathrm{~m}$. The seep deposits from northern Italy and western Washington were previously described by Peckmann et al. (1999, 2002, 2003), Taviani (2011), and Smrzka et al. (2015). 


\section{Analytical methods and results}

Pyrite enclosed in seep carbonates was extracted using the chromium reduction method and is reported in weight percent (wt \%) of the bulk powder (cf. Canfield et al., 1986). Only samples containing less than $0.5 \mathrm{wt} \%$ pyrite were selected for analyses to prevent contamination from potential oxidation of pyrite during carbonate associated sulfate (CAS) extraction (cf. Marenco et al., 2008; Table DR1 and Fig. DR1). Even for those samples that contain $<0.5 \mathrm{wt} \%$ pyrite, it is necessary to estimate the level of sulfate contamination from laboratory procedures. We used Milli-QR water with a low $\delta^{18} \mathrm{O}$ value $(\sim-10 \%)$ for extraction. The $\delta^{18} \mathrm{O}_{\mathrm{CAS}}$ would be consequently lowered if there is a significant contribution of sulfate from sulfide oxidation. We have found this not to be the case in this study (Fig. DR1). Laboratory procedures for the extraction and purification of CAS in bulk carbonates followed the procedures in Peng et al. (2014). These authors reported that extracted CAS can also be severely contaminated by recent atmospheric sulfate, especially for carbonate rock sampled from outcrops in arid to semi-arid climates or heavily polluted regions. None of the samples studied here fall in any of these categories. Nevertheless, a water leaching procedure was applied to the ancient samples to minimize contamination by secondary atmospheric sulfate and secondary sulfate from sulfide oxidation. Large carbonate blocks and pavements were chosen to extract enough CAS and conduct further parallel analyses. In brief, carbonate chips were crushed into small grains using a mortar and pestle. Approximately $100 \mathrm{~g}$ of carbonate powders were immersed 
in $10 \% \mathrm{NaCl}$ solution under constant magnetic stirring at room temperature overnight and repeated three-five times until no sulfate was present in filtered solutions. Rinsed samples were digested in 2-3 M HCl solutions and multiple supernatants were collected until no further fizzing in acid. The solution was then centrifuged, filtered through a $0.2 \mu \mathrm{m}$ filter, and about $5 \mathrm{ml}$ of the solution was then taken from each sample for CAS concentration determination using a Dionex ICS3000 ion chromatograph at Louisiana State University (LSU). Analytical precision for sulfate concentration was typically better than $1 \%$. The remaining solution was acidified before saturated $\mathrm{BaCl}_{2}$ droplets were added. Precipitates were collected after $>12 \mathrm{~h}$ and treated with the DDARP method (Bao, 2006). Acid insoluble residue from CAS extraction was rinsed with deionized water, dried at room temperature and homogenized for pyrite extraction, following the procedure specified in Canfield et al. (1986). The acidified chromium chloride solution was injected into a reaction tube to react with sulfides and produce hydrogen sulfide gas. The resulting sulfide gas was carried by nitrogen gas and then reacted with silver nitrate solution to precipitate silver sulfide. The silver sulfide was rinsed, centrifuged, and dried at $70^{\circ} \mathrm{C}$. The sulfur and oxygen isotopes were analyzed at the Oxy-Anion Stable Isotope Consortium (OASIC) at LSU. Sulfur isotope analyses of extracted $\mathrm{BaSO}_{4}$ and $\mathrm{AgS}_{2}$ was conducted using a Vario MICRO cube elemental analyzer coupled with an Isoprime 100 mass spectrometer. Samples were loaded in a tin capsule and combusted at $1150^{\circ} \mathrm{C}$ in the elemental analyzer. The combustion was catalyzed by $\sim 2 \mathrm{mg}$ of $\mathrm{V}_{2} \mathrm{O}_{5}$ and the resulting $\mathrm{SO}_{2}$ is admitted to the mass spectrometer via a continuous-flow interface. 
The $\delta^{18} \mathrm{O}$ values of $\mathrm{BaSO}_{4}$ were measured via $\mathrm{CO}$ generated by a Thermal Conversion Elemental Analyzer (TCEA) at $1450^{\circ} \mathrm{C}$ coupled with the MAT 253 in continuous-flow mode. The $\delta^{34} \mathrm{~S}$ values are given relative to the Vienna Canyon Diablo troilite $(\mathrm{VCDT}) ; \delta^{18} \mathrm{O}$ values are reported relative to Vienna standard mean ocean water (VSMOW). The precisions for $\delta^{34} \mathrm{~S}$ and $\delta^{18} \mathrm{O}$ determinations were $\pm 0.2 \%$ and $\pm 0.3 \%$, respectively.

Carbonate samples for $\delta^{13} \mathrm{C}$ and $\delta^{18} \mathrm{O}$ analyses were taken from the surfaces of polished slabs using a dental drill. The analyses were conducted using a GV Isoprime II stable isotopic mass spectrometer at Guangzhou Institute of Geochemistry, Chinese Academy of Sciences (GIG, CAS). The $\mathrm{CO}_{2}$ was liberated by reaction with $100 \%$ phosphoric acid at $80^{\circ} \mathrm{C}$. Results are reported in permil (\%o) using the standard $\delta$ notation relative to the Vienna Pee Dee Belemnite (VPDB). Precision was on the order of $0.1 \%$ for $\delta^{13} \mathrm{C}$ and $0.2 \%$ for $\delta^{18} \mathrm{O}$ values.

The ${ }^{87} \mathrm{Sr} /{ }^{86} \mathrm{Sr}$ ratios were determined using a Triton thermal ionization mass spectrometer (TIMS, Thermo Fisher) at GIG, CAS. Powder samples ( $\sim 50 \mathrm{mg})$ were dissolved for 15 minutes in $1.5 \mathrm{~mL} \mathrm{2 \%}$ ultra-pure nitric acid, and then centrifuged. The decantate was mixed with $1.5 \mathrm{~mL} \mathrm{1:1} \mathrm{nitric} \mathrm{acid,} \mathrm{and} \mathrm{then} \mathrm{centrifuged} \mathrm{again.} \mathrm{The}$ ultima decantate was loaded into the column containing Eichrom Sr-specific resin and leached using $4 \mathrm{M}$ nitric acid. The mean value of independent measurements for the SRM 987 is $0.710255(2 \sigma=0.000007, \mathrm{n}=7)$. 


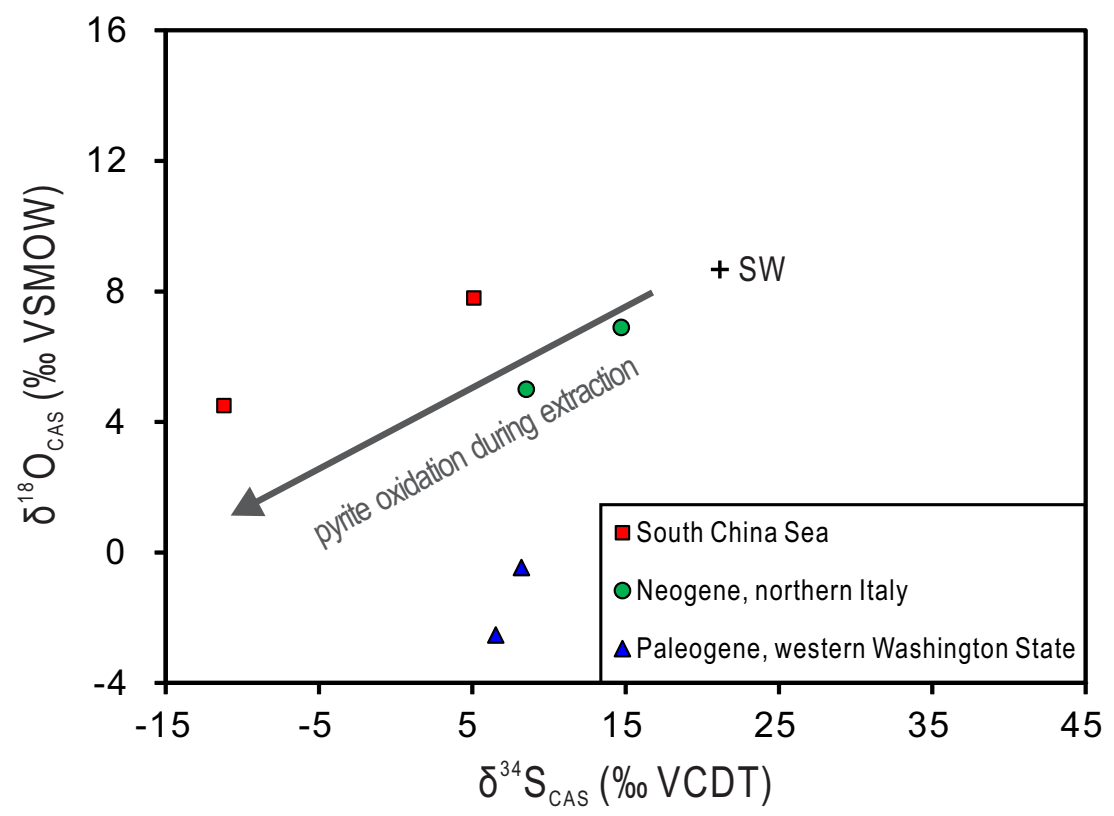

87

Figure DR.1: The $\delta^{34} \mathrm{~S}_{\mathrm{CAS}}$ vs. $\delta^{18} \mathrm{O}_{\mathrm{CAS}}$ of pyrite-rich seep carbonates from modern seafloor (South China Sea) and ancient deposits; the black cross represents the $\delta^{34} \mathrm{~S}$ and $\delta^{18} \mathrm{O}$ values of modern seawater sulfate $(\mathrm{SW})$. Both $\delta^{34} \mathrm{~S}_{\mathrm{CAS}}$ and $\delta^{18} \mathrm{O}_{\mathrm{CAS}}$ values of the depicted samples have been compromised by pyrite oxidation during the CAS extraction process (See Table DR1 for detail). 


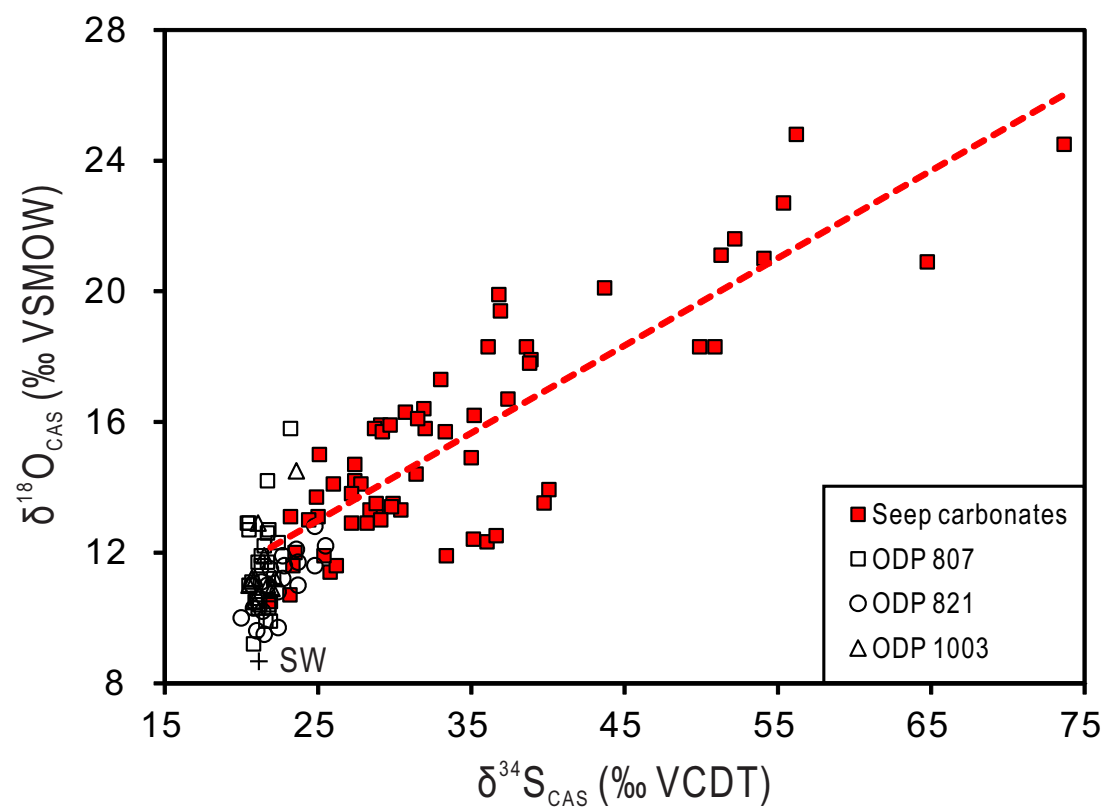

102

103 Figure DR.2: The $\delta^{18} \mathrm{O}_{\mathrm{CAS}}$ versus $\delta^{34} \mathrm{~S}_{\mathrm{CAS}}$ data from modern and ancient authigenic

104 seep carbonates (this study) and from carbonate-rich sediments at three ODP sites

105 (Rennie and Turchyn, 2014): Site 807 (Ontong-Java Plateau; 2815 m water depth),

106 Site 821 (Eastern Australian continental slope; 212 m water depth), Site 1003

107 (Bahama Bank in the Atlantic Ocean; 490 m water depth). VCDT, Vienna Canyon

108 Diablo troilite; VSMOW, Vienna standard mean ocean water. The black cross

109 represents the $\delta^{18} \mathrm{O}$ and $\delta^{34} \mathrm{~S}$ values of modern seawater sulfate.

110

111

112

113

114

115

116 
117 Table DR1: $\delta^{18} \mathrm{O}_{\text {CAS }}$ versus $\delta^{34} \mathrm{~S}_{\mathrm{CAS}}$ data of pyrite-rich modern and ancient seep

118 carbonates.

\begin{tabular}{|c|c|c|c|c|c|}
\hline Samples & Water depth & CAS con. & $\delta^{18} \mathrm{O}_{\mathrm{CAS}}$ & $\delta^{34} \mathrm{~S}_{\mathrm{CAS}}$ & Pyrite content \\
\hline & (m) & (ppm) & (VSMOW) & (VCDT) & $(\%)$ \\
\hline \multicolumn{6}{|l|}{ Modern samples } \\
\hline \multicolumn{6}{|l|}{ South China Sea } \\
\hline TVG $^{\mathrm{a}}$ & 769 & 515 & 4.5 & -11.2 & 1.31 \\
\hline TVG1 $^{\mathrm{a}}$ & 769 & 311 & 7.8 & 5.1 & 1.12 \\
\hline Ancient samples & Ages of deposits & & & & \\
\hline Northern Italy & Neogene & & & & \\
\hline $\operatorname{MarC}^{\mathrm{b}}$ & & 551 & 6.9 & 14.7 & 0.67 \\
\hline Montepetra $1^{\mathrm{c}}$ & & 623 & 5.0 & 8.5 & 0.75 \\
\hline Western Washington State & Paleogene & & & & \\
\hline Wash $10(8)^{\mathrm{d}}$ & & 365 & -0.5 & 8.2 & 0.87 \\
\hline Wash $10(7)^{\mathrm{d}}$ & & 335 & -2.5 & 6.5 & 0.91 \\
\hline
\end{tabular}

$119{ }^{a}$ Described in Tong et al., 2013.

$120{ }^{\mathrm{b}}$ Described in Peckmann et al., 1999.

$121{ }^{\mathrm{c}}$ Described in Taviani, 2011.

$122{ }^{\mathrm{d}}$ Described in Goedert et al., 2003 and Peckmann et al., 2003.

123

124

125

126

127

128

129

130 
131 Table DR2: Geochemical data and dominant carbonate minerals.

\begin{tabular}{|c|c|c|c|c|c|c|c|c|c|c|}
\hline Sample & Water depth & CAS con. & $\delta^{18} \mathrm{O}_{\mathrm{CAS}}$ & $\delta^{34} \mathbf{S}_{\mathrm{CAS}}$ & Py content & $\delta^{34} \mathbf{S}_{\mathbf{P y}}$ & $\delta^{13} \mathbf{C}_{\text {Carb }}$ & $\delta^{18} \mathbf{O}_{\text {Carb }}$ & ${ }^{87} \mathrm{Sr} /{ }^{86} \mathrm{Sr}_{\mathrm{Carb}}$ & \\
\hline & (m) & $(\mathbf{p p m})$ & (VSMOW) & (VCDT) & $(\%)$ & (VCDT) & (VPDB) & (VPDB) & & \\
\hline Modern samples & & & & & & & & & & Dominant carbonate minerals ${ }^{\mathrm{a}}$ \\
\hline \multicolumn{11}{|l|}{ Gulf of Mexico } \\
\hline AT340-1 & 2216 & 454 & 12.9 & 28.2 & 0.28 & 8.0 & -50.8 & 3.0 & 0.708357 & high-Mg calcite \\
\hline AT340-2 & & 118 & 15.8 & 32.0 & 0.01 & -6.2 & -47.5 & 3.4 & 0.709154 & aragonite \\
\hline AT340-3 & & 218 & 13.5 & 29.9 & 0.15 & -0.2 & -48.3 & 2.9 & 0.709167 & aragonite \\
\hline GC140-4 & 287 & 157 & 15.0 & 25.1 & 0.01 & -19.4 & -32.6 & 3.6 & 0.709190 & high-Mg calcite \\
\hline GC140-5 & & 312 & 19.4 & 36.9 & trace & - & -28.4 & 3.3 & 0.709178 & high-Mg calcite \\
\hline GC140/184-1 & 282 & 470 & 12.0 & 23.5 & trace & - & -25.9 & 2.7 & 0.709233 & high-Mg calcite \\
\hline GC140/184-2 & & 101 & 19.9 & 36.8 & 0.01 & -34.7 & -50.8 & 4.1 & 0.709186 & high-Mg calcite \\
\hline GC140/184-3 & & 194 & 13.1 & 23.2 & 0.25 & -12.2 & -31.7 & 3.6 & 0.709192 & high-Mg calcite \\
\hline GC180-1 & 253 & 389 & 13.7 & 24.9 & 0.03 & -8.4 & -16.6 & 3.5 & 0.709225 & high-Mg calcite \\
\hline GC180-2 & & 81 & 15.9 & 29.1 & 0.23 & 7.7 & -28.0 & 2.5 & 0.708713 & high-Mg calcite \\
\hline GC180-3 & & 346 & 15.8 & 28.7 & trace & - & -34.0 & 1.2 & n.a. & high-Mg calcite \\
\hline GC180-4 & & 84 & 14.7 & 27.4 & trace & - & -23.1 & 2.4 & 0.709157 & low-Mg calcite \\
\hline GC180-5 & & 51 & 12.9 & 27.2 & 0.13 & 5.9 & -24.1 & 1.6 & n.a. & low-Mg calcite \\
\hline GC232-1 & 587 & 518 & 14.9 & 35.0 & 0.04 & -9.0 & -21.0 & 3.4 & 0.709179 & high-Mg calcite \\
\hline GC232-2 & & 537 & 14.2 & 27.4 & 0.06 & -7.1 & -16.3 & 3.9 & n.a. & high-Mg calcite \\
\hline GC232-3 & & 112 & 16.2 & 35.2 & 0.02 & -27.0 & -21.5 & 3.4 & 0.709183 & high-Mg calcite \\
\hline GC232-4 & & 565 & 18.3 & 36.1 & 0.23 & -10.6 & -19.6 & 3.5 & 0.709205 & aragonite \\
\hline GC232-5 & & 398 & 18.3 & 38.6 & trace & - & -24.9 & 4.1 & 0.709175 & aragonite \\
\hline GC232-6 & & 317 & 17.3 & 33.0 & 0.15 & -35.6 & -24.9 & 3.3 & 0.709184 & aragonite \\
\hline GC238-1 & 728 & 74 & 24.8 & 56.2 & 0.25 & 4.6 & -47.4 & 2.6 & 0.708817 & low-Mg calcite \\
\hline GC238-2 & & 118 & 21.1 & 51.3 & 0.39 & 4.3 & -43.7 & 3.6 & 0.709081 & low-Mg calcite \\
\hline GC852-1 & 1633 & 157 & 11.4 & 25.8 & 0.03 & 6.9 & -47.6 & 3.3 & 0.709182 & Aragonite \\
\hline
\end{tabular}




\begin{tabular}{|c|c|c|c|c|c|c|c|c|c|c|}
\hline GC852-2 & & 117 & 13.4 & 29.8 & 0.03 & 11.0 & -50.2 & 4.2 & 0.709171 & high-Mg calcite \\
\hline GC852-3 & & 97 & 13.5 & 28.8 & trace & - & -37.7 & 3.7 & n.a. & high-Mg calcite \\
\hline GC852-4 & & 76 & 17.9 & 38.9 & 0.06 & 9.8 & -42.7 & 3.9 & n.a. & high-Mg calcite \\
\hline GC852-5 & & 120 & 13.0 & 29.1 & 0.04 & 13.0 & -46.0 & 3.7 & 0.709190 & high-Mg calcite \\
\hline GC852-6 & & 99 & 15.7 & 33.3 & trace & - & -44.4 & 4.2 & 0.709173 & aragonite \\
\hline GC852-7 & & 138 & 13.3 & 30.4 & 0.03 & 8.2 & -46.9 & 3.7 & n.a. & aragonite \\
\hline GC852-8 & & 67 & 11.6 & 26.2 & 0.03 & 11.8 & -46.6 & 3.0 & n.a. & high-Mg calcite \\
\hline GB260-1 & 420 & 402 & 16.4 & 31.9 & trace & - & -48.8 & 4.0 & n.a. & aragonite \\
\hline GB260-2 & & 86 & 21.0 & 54.1 & 0.07 & 1.8 & -48.2 & 2.2 & 0.708491 & low-Mg calcite \\
\hline GB260-3 & & 78 & 18.3 & 50.9 & 0.03 & 1.7 & -48.0 & 2.2 & 0.708391 & low-Mg calcite \\
\hline GB260-4 & & 49 & 18.3 & 49.9 & 0.03 & 0.3 & -48.6 & 2.2 & 0.708394 & low-Mg calcite \\
\hline GB382-1 & 515 & 254 & 20.1 & 43.7 & trace & - & -31.4 & 4.6 & 0.709173 & high-Mg calcite \\
\hline GB382-2 & & 236 & 13.1 & 25.0 & trace & - & -17.9 & 4.1 & 0.709214 & high-Mg calcite \\
\hline GB382-3 & & 102 & 16.7 & 37.4 & trace & - & -44.4 & 4.4 & 0.709174 & high-Mg calcite \\
\hline GB427-1 & 853 & 169 & 13.0 & 24.4 & trace & - & -42.3 & 4.3 & 0.709890 & high-Mg calcite \\
\hline GB427-2 & & 259 & 16.3 & 30.7 & trace & - & -51.6 & 4.3 & 0.710048 & high-Mg calcite \\
\hline GB427-3 & & 396 & 16.1 & 31.5 & 0.03 & -9.7 & -11.0 & 4.4 & 0.709926 & high-Mg calcite \\
\hline GB427-4 & & 172 & 17.8 & 38.8 & trace & - & -36.5 & 3.6 & 0.709207 & aragonite \\
\hline GB427-5 & & 99 & 10.5 & 21.9 & trace & - & -23.9 & 4.6 & 0.710124 & low-Mg calcite \\
\hline GB427-6 & & 72 & 13.8 & 27.2 & 0.04 & -19.0 & -33.9 & 4.3 & 0.709960 & low-Mg calcite \\
\hline GB427-7 & & 58 & 15.7 & 29.2 & 0.06 & -18.9 & -33.9 & 4.5 & 0.709253 & low-Mg calcite \\
\hline GB427-8 & & 92 & 15.9 & 29.7 & trace & - & -38.1 & 4.1 & n.a. & low-Mg calcite \\
\hline GB647-1 & 950 & 120 & 11.9 & 25.4 & trace & - & -27.4 & 4.3 & 0.708675 & high-Mg calcite \\
\hline GB647-2 & & 234 & 14.1 & 27.8 & 0.09 & -9.9 & -14.8 & 4.0 & n.a. & high-Mg calcite \\
\hline GB647-3 & & 116 & 14.1 & 26.0 & 0.13 & -19.2 & -25.1 & 4.4 & 0.709154 & aragonite \\
\hline MC118-1 & 885 & 207 & 14.4 & 31.4 & 0.47 & -13.3 & -25.6 & 4.2 & 0.709184 & high-Mg calcite \\
\hline MC118-2 & & 223 & 13.1 & 28.8 & 0.17 & -4.2 & -20.1 & 4.4 & 0.709177 & aragonite \\
\hline
\end{tabular}




\begin{tabular}{|c|c|c|c|c|c|c|c|c|c|c|}
\hline MC118-3 & & 241 & 13.3 & 28.4 & 0.40 & -2.2 & -23.1 & 4.8 & 0.709191 & aragonite \\
\hline \multicolumn{11}{|l|}{ Black Sea } \\
\hline Loga 0155 & 120 & 93 & 21.6 & 52.2 & trace & - & -29.2 & 1.8 & n.a. & high-Mg calcite \\
\hline Loga 0110 & 190 & 82 & 24.5 & 73.7 & trace & - & -27.3 & -0.8 & n.a. & high-Mg calcite \\
\hline Ancient samples & Ages of deposits & & & & & & & & & Dominant carbonate minerals \\
\hline Northern Italy & Neogene & & & & & & & & & \\
\hline Marmorito botryoids & & 121 & 22.7 & 55.4 & trace & - & -28.9 & 2.3 & n.a. & aragonite \\
\hline MarES1; Marmorito & & 181 & 11.6 & 23.4 & trace & - & -29.9 & -0.2 & n.a. & high-Mg calcite \\
\hline MarF; Marmorito & & 126 & 10.7 & 23.2 & trace & - & -39.2 & 5.4 & n.a. & high-Mg calcite \\
\hline MarB; Marmorito & & 81 & 20.9 & 64.7 & trace & - & -30.4 & -0.3 & n.a. & high-Mg calcite \\
\hline CaPia; Ca' Piantè & & 174 & 11.9 & 33.4 & trace & - & -46.8 & 3.0 & n.a. & aragonite \\
\hline Western Washington State, USA & Paleogene & & & & & & & & & \\
\hline IV-SSB; Knappton & & 221 & 12.3 & 36.0 & trace & - & -27.6 & 3.3 & n.a. & high-Mg calcite \\
\hline Wash 6(8)-1; SR4 & & 198 & 13.9 & 40.1 & trace & - & -26.6 & 2.4 & n.a. & high-Mg calcite \\
\hline Wash 6(8)-2; SR4 & & 202 & 13.5 & 39.7 & trace & - & -28.5 & 0.9 & n.a. & high-Mg calcite \\
\hline Wash 6(6)-1; SR4 & & 261 & 12.5 & 36.6 & trace & - & -24.7 & 2.8 & n.a. & high-Mg calcite \\
\hline Wash 6(6)-2; SR4 & & 287 & 12.4 & 35.1 & trace & - & -30.1 & 0.1 & n.a. & high-Mg calcite \\
\hline
\end{tabular}

132 CAS, carbonate-associated sulfate; Py, pyrite; Carb, carbonate, n.a., not analyzed.

133 AT, Atwater Valley, GB, Garden Banks, GC, Green Canyon, MC, Mississippi Canyon.

134 a Data from Peckmann et al., 2001; Roberts et al., 2010; Roberts and Feng, 2013; Feng et al., 2014; Feng and Roberts, unpublished data.

135 b Data from Peckmann et al., 1999, 2002; Taviani et al., 2011; Smrzka et al., 2015 


\section{References}

Bao, H.M., 2006, Purifying barite for oxygen isotope measurement by dissolution and reprecipitation in a chelating solution: Analytical Chemistry, v. 78, p. 304-309, doi:10.1021/ac051568z.

Canfield, D.E., Raiswell, R., Westrich, J.T., Reaves, C.M., and Berner, R.A., 1986, The use of chromium reduction in the analysis of reduced inorganic sulfur in sediments and shales: Chemical Geology, v. 54, p. 149-155, doi:10.1016/00092541(86)90078-1.

Feng, D., Birgel, D., Peckmann, J., Roberts, H.H., Joye, S.B., Sassen, R., Liu, X.-L., Hinrichs, K.-U., and Chen, D., 2014, Time integrated variation of sources of fluids and seepage dynamics archived in authigenic carbonates from Gulf of Mexico Gas Hydrate Seafloor Observatory: Chemical Geology, v. 385, p. 129-139, doi:10.1016/j.chemgeo.2014.07.020.

Goedert, J.L., Thiel, V., Schmale, O., Rau, W.W., Michaelis, W., and Peckmann, J., 2003, The Late Eocene 'Whiskey Creek' methane-seep deposit (western Washington State) - Part I: Geology, palaeontology, and molecular geobiology: Facies, v. 48, p. 223-240, doi:10.1007/BF02667541.

Marenco, P.J., Corsetti, F.A., Hammond, D.E., Kaufman, A.J., and Bottjer, D.J., 2008, Oxidation of pyrite during extraction of carbonate associated sulfate: Chemical Geology, v. 247, p. 124-132, doi:10.1016/j.chemgeo.2007.10.006.

Peckmann, J., Goedert, J.L., Heinrichs, T., Hoefs, J., and Reitner, J. 2003, The Late Eocene 'Whiskey Creek' methane-seep deposit (western Washington State) - Part II: Petrology, stable isotopes, and biogeochemistry: Facies, v. 48, p. 241-254, doi:10.1007/BF02667542.

Peckmann, J., Goedert, J.L., Thiel, V., Michaelis, W., and Reitner, J., 2002, A comprehensive approach to the study of methane-seep deposits from the Lincoln 
Creek Formation, western Washington State, USA: Sedimentology, v. 49, p. 855873, doi:10.1046/j.1365-3091.2002.00474.x.

Peckmann, J., Reimer, A., Luth, U., Luth, C., Hansen, B.T., Heinicke, C., Hoefs, J., and Reitner, J., 2001, Methane-derived carbonates and authigenic pyrite from the northwestern Black Sea: Marine Geology, v. 177, p. 129-150, doi:10.1016/S0025$3227(01) 00128-1$.

Peckmann, J., Thiel, V., Michaelis, W., Clari, P., Gaillard, C., Martire, L., and Reitner, J., 1999, Cold seep deposits of Beauvoisin (Oxfordian; southeastern France) and Marmorito (Miocene; northern Italy): Microbially induced authigenic carbonates: International Journal of Earth Sciences, v. 88, p. 60-75, doi:10.1007/s005310050246.

Peng, Y.B., Bao, H.M., Pratt, L.M., Kaufman, A.J., Jiang, G.Q., Boyd, D., Wang, Q.X., Zhou, C.M., Yuan, X.L., Xiao, S.H., and Loyd, S., 2014, Widespread contamination of carbonate-associated sulfate by present-day secondary atmospheric sulfate: Evidence from triple oxygen isotopes: Geology, v. 42, p. 815818, doi:10.1130/G35852.1.

Rennie, V.C.F., and Turchyn, A.V., 2014, The preservation of $\delta^{34} \mathrm{~S}_{\mathrm{SO} 4}$ and $\delta^{18} \mathrm{O}_{\mathrm{SO} 4}$ in carbonate-associated sulfate during marine diagenesis: A 25 Myr test case using marine sediments: Earth and Planetary Science Letters, v. 395, p. 13-23, doi:10.1016/j.eps1.2014.03.025.

Roberts, H.H., and Feng, D., 2013, Carbonate precipitation at Gulf of Mexico hydrocarbon seeps: An overview, in Abrams, M., Aminzadeh, F., Berge, T., Connolly, D., O’Brien, G., eds., Hydrocarbon Seepage: From Source to Surface: SEG/AAPG Special Publication, pp. 43-61, doi:10.1190/1.9781560803119.ch3. Roberts, H.H., Feng, D., and Joye, S.B., 2010, Cold-seep carbonates of the middle and lower continental slope, northern Gulf of Mexico: Deep-Sea Research Part II- 
Topical Studies in Oceanography, v. 57, p. 2040-2054, doi:10.1190/1.9781560803119.ch3.

Smrzka, D., Kraemer, S.M., Zwicker, J., Birgel, D., Fischer, D., Kasten, S., Goedert, J.L., and Peckmann, J., 2015, Constraining silica diagenesis in methane-seep deposits. Palaeogeography, Palaeoclimatology, Palaeoecology, v. 420, p. 13-26, doi:10.1016/j.palaeo.2014.12.007.

Taviani, M., 2011, The deep-sea chemoautotrophic microbial world as experienced by the Mediterranean metazoans through time. in Reitner, J., eds., Advances in Stromatolite Geology. Lecture Notes in Earth Sciences, v. 131, p. 277-295, doi:10.1007/978-3-642-10415-2_18.

Tong, H., Feng, D., Cheng, H., Yang, S., Wang, H., Min, A.G., Edwards, R.L., Chen, Z., and Chen, D., 2013, Authigenic carbonates from seeps on the northern continental slope of the South China Sea: New insights into fluid sources and geochronology: Marine and Petroleum Geology, v. 43, p. 260-271, doi:10.1016/j.marpetgeo.2013.01.011. 